\title{
НАУЧНО-МЕТОДИЧЕСКИЙ ПОДХОД К СОВЕРШЕНСТВОВАНИЮ УПРАВЛЕНИЯ РАЗВИТИЕМ ПРОСТРАНСТВЕННО-ОТРАСЛЕВОЙ СТРУКТУРЫ РЕГИОНА В ЦИФРОВУЮ ЭПОХУ 1
}

\section{SCIENTIFIC METHODICAL APPROACH TO IMPROVING DEVELOPMENT MANAGEMENT OF A REGION'S SPATIAL SECTORAL STRUCTURE IN DIGITAL ERA \\ Ye. Kovaleva S. Pytkina \\ V. Glavatsky}

Summary. In the modern spatial socio-economic development of Russian regions, a significant role is acted by the problem of improving development management of a region's spatial sectoral structure. The article substantiates the scientific methodical approach to improving development management of a regional spatial sectoral structure on based the relationship with systemic and interdisciplinary approaches, as well as with theoretical provisions of transformative management and digital technologies. The study purpose is to substantiate the scientific methodical approach and elaboration tools of the organizational economic model for improving development management of a region's spatial sectoral structure in economy digitalization context.

Keywords: spatial development strategy, spatial sectoral structure, development management, digital technologies, economy digitalization.

\author{
Ковалева Елена Борисовна \\ К.э.н., С.н.С., Пермский филиал Института \\ экономики УрО РАН \\ Пыткина Светлана Алексеевна \\ К.э.н., С.н.С., Пермский филиал Института \\ экономики УрО РАН \\ Главацкий Вадим Борисович \\ К.э.н., дочент, с.н.С., Пермский филиал Института \\ экономики УрО РАН \\ pfie@list.ru
}

Аннотация. В современном пространственном социально-экономическом развитии российских регионов значимую роль играет проблематика совершенствования управления развитием пространственно-отраслевой структуры региона. В статье обосновывается научно-методический подход к совершенствованию управления развитием региональной пространственно-отраслевой структуры на основе взаимосвязи с системным и междисциплинарным подходами, а также с теоретическими положениями преобразующего менеджмента и цифровыми технологиями. Цель исследования — обоснование научно-методического подхода и разработка инструментария организационно-экономической модели совершенствования управления развитием пространственно-отраслевой структуры региона в условиях цифровизации экономики.

Ключевые слова: стратегия пространственного развития, пространственно-отраслевая структура, управление развитием, цифровые технологии, цифровизация экономики.

и методов управления. Именно совершенствование управления развитием пространственно-отраслевой структуры региона должно быть нацелено на обеспечение устойчивого роста экономики территорий, комплексного использования имеющихся в регионе потенциалов и повышения качества жизни местного населения.

По мнению авторов статьи, в российской научно-исследовательской и управленческой практике еще не выработан единый (преобладающий) научно-методический подход к совершенствованию управления развитием региональных пространственно-отрасле-

1 Публикация подготовлена в соответствии с Планом НИР Института экономики УрО РАН на 2021-2023 гг. 
вых структур. К настоящему времени в научной литературе опубликован недостаточный ряд работ, посвященных управлению развитием региональных территорий в условиях цифровизации экономики, что обуславливает повышенное внимание к этой проблеме со стороны экономической науки и актуальность данного исследования.

\section{Цель исслеАования}

Обоснование научно-методического подхода и разработка инструментария организационно-экономической модели совершенствования управления развитием пространственно-отраслевой структуры региона в условиях цифровизации экономики.

\section{Материалы и методы исследования}

В качестве материалов для настоящей статьи были использованы результаты научных исследований в области региональной экономики, пространственного развития территорий, цифровой экономики, менеджмента, а также данные по организационным структурам управления Свердловской областью, Пермским краем и Удмуртской республикой, которые составляют ядро Уральского экономического района. При проведении исследования авторы опирались на междисциплинарный подход, обеспечивавший взаимодействие с положениями таких научных дисциплин как информатика, информационные системы управления, менеджмент, пространственная экономика, цифровые технологии и пр.

Для достижения цели исследования в статье использованы положения системного, комплексного и сетевого научных подходов, применялись методы обобщения научных знаний, сравнительного анализа, организационно-экономического моделирования. Кроме того, были использованы материалы и данные Государственных программ Пермского края на 2021-2023 годы [2], в части внедрения цифровых технологий во все сферы жизни населения региона и развития информационного общества.

\section{Результаты исслеАования}

В настоящее время подходы к совершенствованию управления развитием региональных пространственно-отраслевых структур постоянно претерпевают изменения под влиянием динамичных преобразований внешней и внутренней среды различных сфер социально-экономической жизни регионов. Процесс совершенствования управления развитием пространственно-отраслевой структуры региона является крупной системно-интегрированной проблемой, требующей отдельного обоснования и разработки научно-методического подхода с учетом специфики и характерных особенностей субъекта РФ. Поэтому основу совершенствования управления развитием пространственно-отраслевой структуры региона должен составлять системный научный подход, позволяющий рассматривать региональную пространственно-отраслевую структуру (РПОС) как систему, имеющую вход, процесс, выход, обратную связь и ограничения.

В научной литературе признается, что совершенствование управления РПОС должно осуществляться в системной взаимосвязи с развитием региона, при этом внимание акцентируется на достижении целей и задач пространственного, инновационного и устойчивого социально-экономического развития. Одновременно с этим, управлению РПОС необходимо обеспечить не только позитивную динамику развития экономики региона и рост социального благополучия местного населения, но и рациональное потребление имеющихся в регионе потенциалов, их сбалансированное, расширенное и интенсивное воспроизводство [3, $4]$.

В связи с этим, при формировании научно-методического подхода к совершенствованию региональной пространственно-отраслевой структуры авторы статьи среди основных принципов системного научного подхода выделили следующие:

1. Системность - принцип обладания РПОС основными признаками системы;

2. Целостность - принцип рассматривает пространственно-отраслевую структуру региона как единую целую систему и одновременно как подсистему для вышестоящего уровня управления;

3. Иерархичность - принцип обеспечивает уровневое и функциональное построение РПОС на основе подчинения структурных элементов низшего уровня структурам высшего уровня;

4. Структурность - принцип позволяет осуществлять аналитические расчеты по оценке функционирования элементов РПОС и их взаимосвязи в рамках организационной структуры управления;

5. Множественность - принцип предоставляет возможность применять множество целенаправленных организационно-экономических, математических и кибернетических моделей для описания отдельных структурных элементов и функций, а также РПОС в целом.

Научно-методический подход авторов к совершенствованию управления развитием региональной пространственно-отраслевой структуры опирается так же на теоретические положения, модели и алгоритмы 
расчетов целой группы научных дисциплин, среди которых информатика, информационные системы управления, пространственная экономика, преобразующий менеджмент, цифровые технологии и пр. Сопряжение теории и практики управления РПОС с рядом научных дисциплин формирует междисциплинарный подход к обоснованию научно-методического подхода к совершенствованию управления развитием пространственно-отраслевой структуры региона.

При реализации междисциплинарного подхода к совершенствованию управления развитием региональной пространственно-отраслевой структуры в условиях цифровизации значимую роль играет взаимосвязь управления с преобразующим менеджментом и цифровыми технологиями (информационно-коммуникационными и управленческими), что обуславливает переход управления РПОС на более высокий эффективный уровень. По мнению авторов, ключевой аргументацией применения цифровых технологий в управлении развитием пространственно-отраслевой структуры являются уникальные свойства, которыми эти технологии обладают. К таким можно отнести следующие свойства:

- инновационность - высокая скорость, качество и надежность передачи, хранения и обработки цифровых сигналов с сохранением точности данных для принятия управленческих решений;

- критериальность - мгновенный поиск релевантной информации по заданным критериям с соблюдением стандарта информационной безопасности;

- адаптивность - гибкость цифровых технологий в работе с широким диапазоном типов информации по запросу пользователей;

- функциональность - простота использования, удобство и логичность интерфейсов, различные форматы данных;

- интегрируемость - построение многоуровневых сетевых интегрированных автоматизированных систем управления, обмен данными между цифровыми устройствами, одновременный многоканальный доступ пользователей к оцифрованным данным;

- экономичность - минимальные предельные издержки передачи цифровых сигналов в рамках интегрированной сетевой структуры.

В целом свойства цифровых технологий и их важнейшие преимущества для потенциальных пользователей заключаются прежде всего в том, что они обеспечивают $[5,6]$ :

- одновременный доступ многих пользователей к оперативной и архивной информации;

- практически мгновенный поиск больших объемов релевантной информации по заданным критериям;
- быстрый доступ к оцифрованным данным, которые могут храниться в архиве или в облачном онлайн-хранилище (последний вариант используется для хранения некритически важных данных).

Помимо применения цифровых технологий, как важный аспект формирования научно-методического подхода к совершенствованию управления развитием РПОС следует рассматривать взаимосвязь управления РПОС с теоретическими положениями преобразующего менеджмента, поскольку такой менеджмент предполагает совершенствование управления с качественными изменениями, то есть с возникновением новых устойчивых структурных составляющих элементов, связей и зависимостей. Другими словами, процесс совершенствования связан с преобразованием структуры системы управления [7]. Здесь необходимо понимать процесс закономерного перехода системы управления с одного качественного уровня на другой, обеспечивающий повышение конкурентности системы управления РПОС [8].

Проведенное авторами исследование глубины организационно-функциональных изменений в управлении пространственно-отраслевыми структурами Пермского края и Свердловской области, а также анализ зарубежной и отечественной литературы позволяет выделить пять уровней совершенствования управления РПОС:

- минимальный (в рамках сложившейся организационной структуры управления уточняются и меняются только полномочия и функции);

- умеренный (совершенствование технологий коммуникации, обработки и хранения информации, мониторинга; внесение изменений в решение функциональных задач с применением информационных технологий и т.п.);

- средний (внедрение управленческих инноваций, связанных с расширением автоматизации отдельных функциональных задач управленческой деятельности; модернизация вычислительной техники, программного обеспечения и др.; формирование единого информационного пространства);

- высокий (переход на современные технологические платформы автоматизации управленческой деятельности, внедрение новых схем в систему информационно-коммуникационных технологий; подбор профессионально подготовленного кадрового обеспечения управления регионом и др.);

- максимальный (существенная перестройка организационных, функциональных, технических, программных аспектов управленческой дея- 


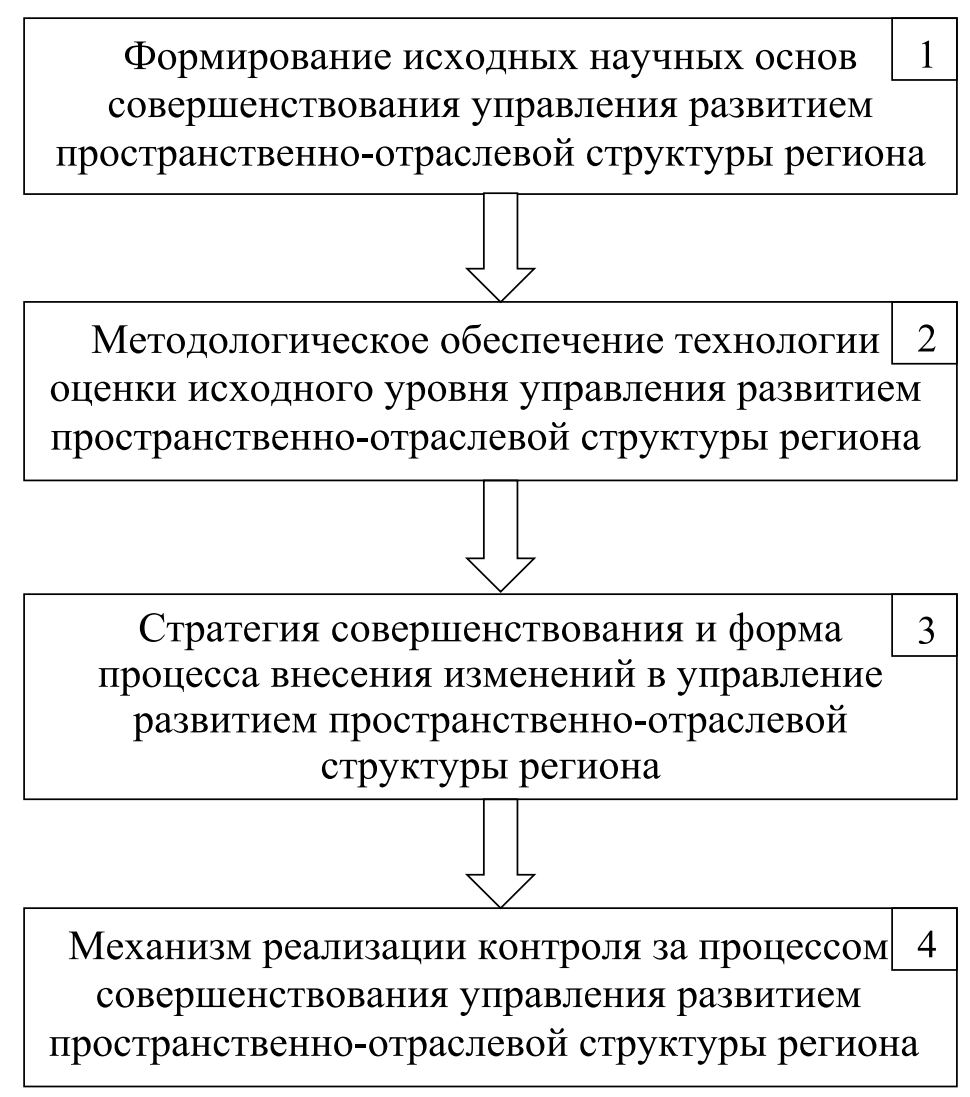

Рис. 1. Организационно-экономическая модель совершенствования управления развитием пространственно-отраслевой структуры региона

тельности; переход на использование цифровой инфраструктуры и цифровых технологий в управлении; внедрение инновационной модели управленческой деятельности, способной решать сложные управленческие задачи, отражающие динамичные изменения в окружающей среде).

Следует отметить, что в настоящее время в российских регионах преобладает эволюционное направление совершенствования управления развитием пространственно-отраслевых структур с соблюдением традиционных организационных структур управления и подходов к автоматизации функциональных задач управленческой деятельности, что не позволяет своевременно и адекватно реагировать на возникающие отклонения и проблемы в социально-экономических процессах, происходящих в регионах РФ. В связи с чем можно сделать вывод, что несмотря на достижение определенной стабильности в управлении развитием пространственно-отраслевой структурой, регионы не в полной мере используют потенциальные возможности цифровизации экономики, что снижает научно-технический уровень управления РПОС.
Вышеизложенное позволяет авторам предложить научно-методический подход к совершенствованию управления развитием региональной пространственно-отраслевой структуры, основные положения которого базируются на четырех ключевых этапах организационно-экономической модели, изображенной на рисунке 1.

Первый этап «Формирование исходных научных основ совершенствования управления развитием пространственно-отраслевой структуры региона», в котором закладывается методологический базис (научные подходы, принципы и методы) совершенствования управления РПОС, является основополагающим и определяющим в организационно-экономической модели. К ключевым научным подходам совершенствования управления РПОС следует отнести подходы:

- инновационный (нововведения в систему управления);

- информационный (новые формы и способы получения релевантной информации);

- функциональный (совершенствование управленческих бизнес-процессов);

- проблемно-ориентированный (выявление и решение управленческих проблем) и пр. 
Совершенствование управления развитием РПОС обуславливает необходимость применения совокупности следующих принципов: прямоточность (кратчайший путь получения релевантной информации), адаптивность (реакция на изменение факторов окружающей среды), связанность (оптимизация вертикальных и горизонтальных связей в управлении), специализация (функциональное разделение управленческой деятельности), эффективность (оценка затрат на содержание аппарата управления во взаимосвязи с результатами управленческой деятельности по социально-экономическому развитию региона).

Научные подходы и принципы совершенствования управления развитием РПОС неразрывно связаны с методами управления, которые по своему назначению и содержанию классифицируются в три группы: организационно-административные, экономические (соизмерение затрат с результатами управленческой деятельности) и социально-психологические (сохранение морально-психологического климата в коллективе при достижении целей управленческой деятельности).

Второй этап «Методическое обеспечение технологии оценки исходного уровня управления развитием пространственно-отраслевой структуры региона» выполняет сложную аналитическую задачу. Сложность ее состоит в том, что технология оценки исходного уровня управления РПОС заключается в необходимости: во-первых, определить результаты деятельности управления по достижению целей социально-экономического развития региона; во-вторых, комплексно оценить качество функционирования системы управления регионом; в-третьих, оценка качества должна позволить проанализировать эффективность функционирования каждого элемента управления и его в целом.

Кроме того, технология оценки исходного уровня управления развитием пространственно-отраслевой структуры должна определить: организационно-функциональную структуру управления РПОС; группы аналитических показателей, характеризующих достигнутый научно-технический уровень управления РПОС; уровень научной организации труда в аппарате управления регионом и профессиональную подготовку к управленческой деятельности.

Третий этап «Стратегия совершенствования и форма процесса внесения изменений в управление развитием пространственно-отраслевой структуры региона» является сложным, поскольку характеризуется многоаспектностью и многовариантностью мероприятий для их отбора по включению в планы, проекты и программы совершенствования управления развитием РПОС. На данном этапе в стратегии совершен- ствования управления развитием РПОС внимание акцентируется на временном горизонте внесения изменений (краткосрочный период - до 3 месяцев, среднесрочный - 0,5 года и долгосрочный с переподготовкой управленцев - до 1 года). Формой процесса внесения изменений в управление развитием пространственно-отраслевой структуры в зависимости от цели изменений и режима управления являются план, проект и программа. Использование планов для процесса внесения изменений в управление РПОС обуславливается сложными и многоцелевыми задачами совершенствования, требующими координационный режим управления. Для решения одноцелевых задач и одноцелевого режима управления используются проекты и программы.

Следует отметить, что внесение сложных изменений в управление РПОС может выходить за рамки долгосрочного периода (1 год) и полностью быть законченным к окончанию инновационного периода.

Четвертый этап «Механизм реализации контроля за процессом совершенствования управления развитием пространственно-отраслевой структуры региона» является конечным результатом проведения изменений в управлении РПОС, который имеет четкий регламент осуществления мониторинга целевого управления развитием пространственно-отраслевой структуры региона.

Представленный научно-методический подход к совершенствованию управления развитием региональной пространственно-отраслевой структуры позволяет детально изучить возможности системного и междисциплинарного подходов с акцентированным вниманием на свойствах цифровых технологий и обосновать на этой основе организационно-экономическую модель совершенствования управления развитием РПОС с учетом теоретико-методологических основ преобразующего менеджмента и специфики управленческой деятельности регионального менеджмента.

\section{Зак^ючение}

В проведенном исследовании выявлено, что подходы к совершенствованию управления развитием пространственно-отраслевых структур постоянно претерпевают изменения под влиянием динамичных преобразований внешней и внутренней среды различных сфер социально-экономической жизни регионов. Представленный в статье научно-методический подход к совершенствованию управления развитием пространственно-отраслевой структуры региона опирается на взаимосвязи с системным и междисциплинарным подходами, а также с теоретическими положениями 
преобразующего менеджмента и цифровыми технологиями.

На основе предложенного научно-методического подхода разработана организационно-экономическая модель совершенствования управления развитием РПОС состоящей из четырех этапов, имеющих цель, задачи и системную проработку, что служит научно-методической основой для разработки стратегии совер- шенствования и формы процесса внесения изменений в управление развитием РПОС.

Дальнейшее развитие данного исследования предполагает уточнение теоретической базы для обоснования, отбора и реализации планов, программ и проектов совершенствования управления развитием РПОС на базе функциональных бизнес-процессов системы программно-проектного менеджмента.

\section{ЛИТЕРАТУРА}

1. Стратегия пространственного развития Российской Федерации на период до 2025 года (утв. распоряжением Правительства РФ от 13.02.2019 № 207-р, ред. от 23.03.2021). Консультант Плюс. URL: http://consultant.ru/document/cons_doc_LAW_318094 (дата обращения: 20.05.2021).

2. Государственные программы Пермского края 2021-2023. URL: https://mfin.permkrai.ru/upload/pages/1884/dat_160620053988.pdf (дата 0бращения: 20.05.2021).

3. Конкурентоспособность социально-экономических систем: вызовы нового времени / под науч. ред. А.И. Татаркина, В.В. Криворотова. 一 М.: Экономика, 2014. 466 с.

4. Пыткин А.Н. Формирование направлений и приоритетов стратегического развития пространственно-отраслевой структуры региона с учетом информатизации российской экономики // Экономика, предпринимательство и право. — 2021. — Т. 11.— № 6. — doi: 10.18334/epp.11.6.112198.

5. Маркова В.Д. Цифровая экономика.— М.: Инфра-М, 2018. 186 с.

6. АНО «Цифровая экономика». Официальный сайт. URL: https://data-economy.ru (дата обращения: 01.06.2021).

7. Гительман Л.Д. Преобразующий менеджмент: лидерам реорганизации и консультантам по управлению.— М.: Дело, 1999. 496 с.

8. Буторин С.Н. Формирование и развитие конкурентной системы управления аграрными предприятиями. — Екатеринбург: ИЭ Ур0 РАН, 2016. 203 с.

( ) Ковалева Елена Борисовна, Пыткина Светлана Алексеевна, Главацкий Вадим Борисович ( pfie@list.ru ).

Журнал «Современная наука: актуальные проблемы теории и практики»

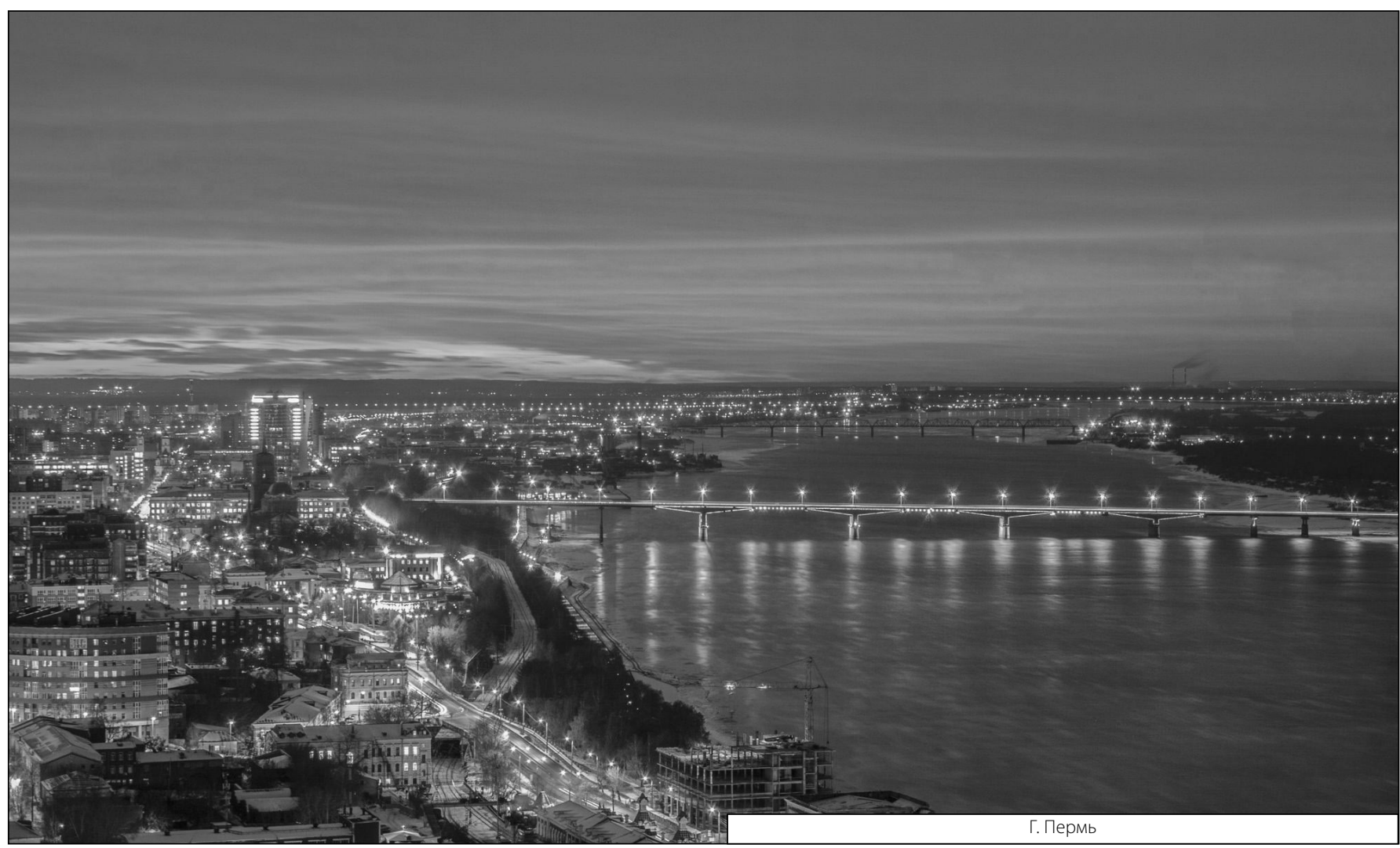

